INPLASY

PROTOCOL

To cite: Liu et al. Prognostic value of long non-coding RNA PVT1 as a potential biomarker in osteosarcoma: A protocol for systematic review and meta-analysis. Inplasy protocol 202090050. doi:

10.37766/inplasy2020.9.0050

Received: 12 September 2020

Published: 12 September 2020

Corresponding author: Hong Sun

sunhong002@126.com

Author Affiliation:

Department of Orthopaedics, the Affiliated Hospital of Guizhou Medical University

Support: Grant NO. gzwjkj2019-1-134.

Review Stage at time of this submission: Preliminary searches.

Conflicts of interest:

The authors declare that there is no competing interest.

\section{Prognostic value of long non-coding RNA PVT1 as a potential biomarker in osteosarcoma: A protocol for systematic review and meta-analysis}

Liu, M1' Liang, TH2; Wang, FY3; Yang, $\mathrm{H}^{4}$; Ning, $\mathrm{X}^{5}$; Sun, $\mathrm{H}^{6}$.

Review question / Objective: Is the expression level of IncRNA PVT1 associated with the prognosis of patients with osteosarcoma?

Condition being studied: Long noncoding RNA (IncRNA) PVT1 has been found aberrantly expressed in osteosarcoma. Upregulated PVT1 expression accelerates the proliferation, invasion, and migration of osteosarcoma cells. Moreover, several studies reported that high level of PVT1 is associated with poor clinical outcomes in patients with osteosarcoma. However, the prognostic value of IncRNA PVT1 in forecasting the clinical outcomes in patients undergoing osteosarcoma still largely unknown. Thus, the aim of this study is to systematically review the available literature to explore the correlation between PVT1 expression and prognosis in patients with OS.

INPLASY registration number: This protocol was registered with the International Platform of Registered Systematic Review and Meta-Analysis Protocols (INPLASY) on 12 September 2020 and was last updated on 12 September 2020 (registration number INPLASY202090050).

\title{
INTRODUCTION
}

Review question / Objective: Is the expression level of IncRNA PVT1 associated with the prognosis of patients with osteosarcoma?
Condition being studied: Long noncoding RNA (IncRNA) PVT1 has been found aberrantly expressed in osteosarcoma. Upregulated PVT1 expression accelerates the proliferation, invasion, and migration of 
osteosarcoma cells. Moreover, several studies reported that high level of PVT1 is associated with poor clinical outcomes in patients with osteosarcoma. However, the prognostic value of IncRNA PVT1 in forecasting the clinical outcomes in patients undergoing osteosarcoma still largely unknown. Thus, the aim of this study is to systematically review the available literature to explore the correlation between PVT1 expression and prognosis in patients with OS.

\section{METHODS}

Search strategy: Plasmacytoma variant translocation 1 or PVT1 and osteosarcoma.

Participant or population: Patients who are diagnosed with osteosarcoma.

Intervention: The OS patients in intervention group are with high expression level of PVT1.

Comparator: The OS patients in control group are with low expression level of PVT1.

Study designs to be included: (i) studies exploring the correlation between PVT1 expression and the prognosis of patients with osteosarcoma; (ii) studies in which patients were divided into two groups according to their expression level of PVT1; (iii) studies in which clinical parameters such as tumor size, clinical stage and distant metastasis were described; (iiii) studies which provided available HRs and $95 \%$ Cls of OS or survival curves for calculating.

Eligibility criteria: The eligibility criteria are summarized using PICOS approach (patients, intervention, comparisons, outcome, and study design type).

Information sources: Comprehensive search will be performed by using PubMed, Embase, Web of Science, Cochrane Library, Wanfang database and CNKI from their dates of inception up to August 31, 2020.
Main outcome(s): The primary outcome is the correlation between PVT1 expression and overall survival of patients with osteosarcoma.

Additional outcome(s): The secondary outcomes are the correlations between PVT1 expression and clinicopathological characteristics, including age and gender, tumor size, tumor anatomic location, clinical stage, and distant metastasis.

Data management: All data will be extracted from enrolled article contexts, tables, and figures by two independent reviewers. The following data will be extracted: surname of the first author, publication date, country, participants' age and gender, sample size, tumor size, tumor anatomic location, clinical stage, distant metastasis, and OS.

Quality assessment / Risk of bias analysis: All eligible studies were non-randomized controlled studies. Study quality was assessed using the Newcastle-Ottawa Scale (NOS) and a NOS score of $\geq 6$ was considered high quality.

Strategy of data synthesis: All statistical analyses will be analyzed by using Stata12 (Stata Corp, College Station, Texas). HRs and ORs will be adopted to compare OS and clinicopathologic parameters respectively. Heterogeneity among studies will be assessed using Chi-square $Q$ test and 12 statistic. The random-effect model or fixed effect model will be used to calculate pooled results. Publication bias will be measured by using Begg's funnel plots and Harbord's modified test. $P<0.05$ is considered statistically significant.

Subgroup analysis: If necessary, we will explore sources of apparent heterogeneity based on the differences in study characteristics, study quality and outcomes.

Sensibility analysis: Not applicable.

Language: English.

Country(ies) involved: China. 
Keywords: Long non-coding RNA, PVT1; Osteosarcoma; Prognosis; systematic review and Meta-analysis.

Contributions of each author:

Author 1 - Miao Liu - Data curation and funding acquisition.

Author 2 - Tihong Liang - Data curation.

Author 3 - Fengyan Wang - Formal analysis.

Author 4 - Hua Yang - Methodology.

Author 5 - Xu Ning - Writing-review \& editing.

Author 6 - Hong Sun - Conceptualization and writing review and editing. 